\title{
Habilidades Sociais e Vivência Acadêmica de Estudantes Universitários
}

\author{
Adriana Benevides Soares* \\ Universidade Salgado de Oliveira, Niterói, RJ, Brasil \\ Universidade do Estado do Rio de Janeiro, Rio de Janeiro, RJ, Brasil \\ Luciana Mourão \\ Universidade Salgado de Oliveira, Niterói, RJ, Brasil \\ Acácia Aparecida Angeli dos Santos \\ Universidade São Francisco, Itatiba, SP, Brasil
}

Thatiana Valory dos Santos Mello

Universidade Estácio de Sá, Niterói, RJ, Brasil

\begin{abstract}
RESUMO
O estudo objetivou caracterizar os universitários quanto ao repertório de habilidades sociais e à vivência acadêmica, examinando a correlação entre tais variáveis e comparando as diferenças por gênero e curso. Participaram 202 alunos de Informática e Psicologia, com idades entre 16 e 49 anos $(M=22,84 ; D P=5,92)$, sendo $75,6 \%(\mathrm{n}=146)$ homens, que responderam ao Questionário de Vivência Acadêmica - reduzido, Escala de Avaliação da Vida Acadêmica e Inventário de Habilidades Sociais. Os resultados indicaram percepção moderada da vivência acadêmica e do repertório de habilidades sociais e correlações de baixa magnitude entre essas variáveis. Diferenças entre os cursos e entre os sexos foram identificadas. Os achados são discutidos com base na literatura e novas pesquisas são sugeridas.
\end{abstract}

Palavras-chave: habilidades sociais; universitários; vivência acadêmica.

\section{ABSTRACT \\ Social Skills and Academic Integration of College Students}

The study aimed to describe the students regarding social skills and academic integration, discussing the correlation between these variables and comparing possible differences by gender and course. The sample was composed of 202 undergraduates from two different courses, ranging from 16 to 49 years $(\mathrm{M}=22.84 ; \mathrm{SD}=5.92)$, of which $75.6 \%(\mathrm{n}=146)$ were male. They responded to three instruments: Questionário de Vivência Acadêmica - reduzido, Escala de Avaliação da Vida Acadêmica and Inventário de Habilidades Sociais. Results showed intermediate levels of academic integration and social skills and correlations with low magnitude between these variables. Differences between courses and gender were identified. The findings are discussed based on the literature, and new investigations are suggested.

Keywords: academic experience; social skills; undergraduate students.

Na conjuntura atual, a massificação do Ensino Superior é uma realidade inquestionável, com expressivo aumento do número de estudantes (Almeida, Marinho-Araújo, Amaral, \& Dias, 2012). Em consequência disso, também tem se ampliado o interesse por estudar o público universitário e alguns aspectos a ele relacionados, tais como suas vivências acadêmicas e habilidades sociais. Assim, os estudos sobre o estudante universitário e suas vivências no Ensino Superior têm sido crescentes desde o surgimento dos primeiros modelos teóricos explicativos (Terenzini, Lorang, \& Pascarella, 1981; Tinto, 1975) e de autores subsequentes. Vários estudos foram feitos para compreender o problema da evasão de alunos em Portugal (Almeida, Soares, \& Ferreira, 1999; Azevedo \& Faria, 2001; Pires, Almeida \& Ferreira, 2000, Soares, Almeida,

* Endereço para correspondência: Adriana Benevides Soares - adribenevides@gmail.com 
Diniz, \& Guisande, 2006) e no Brasil (Igue, Bariani, \& Milanesi, 2008; Santos, Suehiro, Oliveira, Cunha, \& Carrilho, 2006; Soares, Poubel, \& Mello, 2009).

Sobre o desenvolvimento dos universitários e suas vivências no meio acadêmico, Almeida et al. (1999) destacam que ele depende de circunstâncias objetivas, mas também dos aspectos individuais do aluno, particularmente da capacidade adaptativa de cada um, do seu grau de vulnerabilidade e da capacidade de enfrentamento de situações estressoras tais como avaliações, relações com autoridades, dentre outras. Assim, as vivências acadêmicas possuem singularidades que evidenciam que apenas o estabelecimento de um ambiente acadêmico e social apropriado à integração e ao desenvolvimento do aluno, embora desejável, não é suficiente se os alunos não o perceberem como um contexto propício que lhes oferece oportunidades de experimentação ativa.

Ao abordar o tema das vivências universitárias, autores como Ferreira, Almeida e Soares (2001) esclarecem que o ambiente acadêmico se estabelece como um contexto que influi no desenvolvimento do estudante. Os estudiosos da área têm mostrado, por meio de suas pesquisas, que um ambiente que possibilite bem-estar acadêmico aos estudantes, caracterizado por um contexto de mais confiança pode ser desencadeador do desenvolvimento do aluno do Ensino Superior, ajudando em sua adaptação e seu sucesso acadêmico (Cunha \& Carrilho, 2005; Granado, Santos, Almeida, Soares \& Guisande, 2005). O oferecimento de condições de incentivo à integração à vida universitária, como enfatizam Polydoro, Primi, Serpa, Zaroni e Pombal (2001), principalmente no primeiro ano, pode ser decisiva na manutenção ou exclusão do aluno do sistema superior de ensino.

Considerando o acesso ampliado às universidades, ocorrido nos últimos anos, Zago (2006) alerta que, principalmente para o aluno advindo de camadas sociais menos favorecidas, não faz uma verdadeira escolha pelo curso universitário, visto que em grande parte das vezes ingressa na Instituição de Ensino Superior IES que lhe oferece condições de frequentá-lo (por exemplo, mais próxima, mais acessível financeiramente, processo seletivo mais fácil etc.). Assim, ele procura se adaptar, levando em conta suas capacidades e sua condição de vida e trabalho, visando minimizar a possibilidade de ficar de fora do contexto universitário. Estudiosos têm asseverado que o pro- cesso de acomodação à vida universitária pode ser agravado pelos conflitos e mudanças inerentes à passagem da idade adolescente para a idade adulta (Teixeira, Dias, Wottrich, \& Oliveira, 2008), mas que diversos aspectos característicos dessa fase interferem, sejam eles atribuídos ao indivíduo ou ao ambiente em que se inserem (Almeida \& Soares, 2003; Granado et al., 2005).

O estudo de Baker e Siryk (1989) aponta quatro fatores relacionados a adaptação ao Ensino Superior que se referem ao ajustamento acadêmico, ao ajustamento relacional-social, ao ajustamento pessoal-emocional e ao comprometimento com a instituição e aderência ao curso. Soares et al. (2006) apontam fundamentalmente como fator de impacto no ajustamento ao Ensino Superior a autonomia emocional e instrumental, assim como o investimento na vida acadêmica, sendo que estes fatores variam em função do curso e do gênero. A avaliação da vida acadêmica tem sido realizada com base nas teorias desenvolvimentistas e modelos de impacto, referindo-se a um processo multifacetado que envolve aspectos pessoais; como a percepção de autonomia e competência pessoal, bem estar físico e psicológico, auto confiança; aspectos interacionais que se referem ao relacionamento com colegas e professores; e aspectos institucionais relativos a adaptação ao curso e a instituição e ao desenvolvimento da carreira (Tinto, 1975; Terenzini, Lorang, \& Pascarella, 1981; Pascarela \& Terenzini, 2005).

Sob esse enfoque, vários estudos empíricos têm demonstrado a relevância de se entender a integração e a adaptação do estudante à universidade. Alguns deles, realizados no Brasil, serão aqui trazidos, a fim de ilustrar o potencial de contribuição que seus resultados propiciam para a compreensão do fenômeno estudado. Na última década foram desenvolvidos e adaptados no Brasil instrumentos com evidências de validade psicométrica que permitem investigar as vivências acadêmicas, entre os quais se destacam o Questionário de Vivência Acadêmica - reduzido (QVA-r) (Granado et al., 2005) e a Escala de Avaliação da Vida Acadêmica (EAVA) (Vendramini et al., 2004) em que os escores mais altos indicam maior adaptação ao Ensino Superior.

Com o propósito de compreender como ocorrem as vivências do aluno à universidade, Teixeira, Castro e Piccolo (2007) propuseram-se a avaliar algumas das variáveis relevantes nesse processo. Dessa forma, 
usando o Questionário de Vivências Acadêmicas, em sua versão reduzida (QVA-r), investigaram as cinco grandes áreas de vivências no âmbito universitário, englobadas pelo instrumento, a saber, pessoal, interpessoal, carreira, estudo e institucional. Os resultados obtidos revelaram médias acima do ponto médio (valor 3), sugerindo que a maioria deles identificava-se como razoavelmente adaptados ao contexto da universidade. As médias mais elevadas foram obtidas nas dimensões carreira e interpessoal, revelando satisfação com o curso e com as relações pessoais estabelecidas no âmbito da universidade. Por sua vez, a dimensão pessoal apresentou a menor média, sugerindo que o ajustamento pessoal e o sentimento de bem-estar dos estudantes não são tão altos, quanto a satisfação com o curso e com as relações interpessoais. Os autores ressaltam que isso não pode ser encarado como evidência de que apresentavam dificuldades de ajuste pessoal, visto que a média nessa subescala ainda ficou acima do ponto médio.

Igue, Bariani e Milanesi (2008), também utilizando o QVA-r, identificaram em 203 discentes de psicologia de universidade confessional do interior do estado de São Paulo, que as relações interpessoais estabelecidas por eles se relacionam de maneira significativa com a IES que frequentam. Similarmente ao observado no estudo de Teixeira et al. (2008), a pontuação média em todas as subescalas ficou acima do ponto médio, sendo as relativas ao bem-estar pessoal mais negativas em relação às demais. No entanto, segundo as percepções dos estudantes, eles conseguiam estudar de forma equivalente aos colegas, que estão satisfeitos com sua carreira e seu curso e que conheciam mal os recursos que a instituição oferecia.

Com escopo mais amplo, o Inventário de Habilidades Sociais (IHS) também tem sido usado com a população universitária. No presente estudo esses três instrumentos foram empregados, com a pressuposição de que o nível de habilidades sociais poderia favorecer a integração dos estudantes com o mundo acadêmico, modificando a percepção dos mesmos sobre suas vivências acadêmicas, tal como enfatizado pelos trabalhos apresentados a seguir.

Segundo Bolsoni-Silva, Loureiro, Rosa e de Oliveira (2010), as habilidades sociais podem ser responsáveis por um processo social adaptativo saudável que asseguram o bem-estar físico e psicológico do estudante. Estudantes socialmente competentes deveriam apresentar processos de ajustamento a contextos desconhecidos mais rápidos e eficazes do que os nãohabilidosos. Vários estudos empíricos a fim de buscar caracterizar os estudantes universitários e suas interações interpessoais utilizaram o Inventário de Habilidades Sociais (IHS, Del Prette, 2001). Dentre eles, Barreto, Pierre, Del Prette e Del Prette (2004) compararam o repertório de habilidades sociais de estudantes universitários de homens e mulheres e verificaram diferenças significativas no que concerne à assertividade, expressão de sentimentos positivos e controle da agressividade sendo os participantes do sexo masculino com escores maiores do que os do sexo feminino. Bartholomeu, Nunes e Machado (2008) correlacionaram o repertório de habilidades sociais e traços de personalidade e encontraram que alguns aspectos da personalidade tais como amabilidade, pró sociabilidade e confiança relacionam com fatores das habilidades sociais tais como autoafirmação; autoafirmação e autocontrole; enfrentamento, autoafirmação e autocontrole, respectivamente.

Alguns outros estudos (Baldez; 2009; Gerk \& Cunha, 2006; Soares, Poubel, \& Melo, 2009; Soares et al., 2013) buscaram verificar a associação entre adaptação à universidade e o conjunto de habilidades sociais utilizando os mesmos instrumentos propostos neste estudo. Gerk e Cunha (2006), por exemplo, em estudo com 99 estudantes de ciências exatas de uma instituição em que a exigência acadêmica para entrada e manutenção dos alunos é reconhecidamente socialmente como alta, identificaram um grande número de correlações entre as medidas de habilidades sociais e de vivências acadêmicas. As correlações mais fortes aconteceram entre o escore total do inventário de habilidades sociais e as subescalas do questionário de vivências acadêmicas: relacionamento com os colegas; autonomia pessoal; autoconfiança; percepção pessoal de competências cognitivas.

No estudo de Ferreira, Almeida e Soares (2001) foram encontrados resultados significativamente superiores para os homens no que concerne a bem-estar psicológico, autoconfiança, percepção de competências pessoais, envolvimento em atividades extracurriculares, bases de conhecimento para o curso, realização de exames. Enquanto para as mulheres maiores resultados foram identificados no relacionamento com a família e nos métodos de estudo. Para os alunos do curso de Psicologia/Educação foram identificados maiores escores para relacionamento com os professo- 
res e adaptação ao curso em relação aos cursos de Economia, Ciências Sociais, Ciências, Engenharias, Letras e Ciências Humanas.

No estudo de Soares et al. (2013) foram encontrados resultados para os participantes do gênero masculino superiores no que diz respeito as habilidades sociais de enfrentamento e autoafirmação com risco, porém, no que se relaciona à autoafirmação na expressão de afeto positivo o gênero feminino obteve escores superiores ao masculino. Quanto às vivências acadêmicas na dimensão Interpessoal e Estudo, o gênero feminino obteve escores significativamente superiores ao masculino.

Frente às considerações concernentes aos fenômenos particularmente importantes para as vivências acadêmicas no Ensino Superior, o presente estudo se propôs a caracterizar os universitários com relação ao repertório de habilidades sociais e às vivências acadêmicas, examinar a relação entre esses construtos e comparar diferenças por gênero e curso. A fundamentação teórica permite que se estabeleça como hipótese a existência de correlações significativas entre os escores em habilidades sociais e de vivências acadêmicas.

\section{MÉTODO}

\section{Participantes}

Este estudo foi realizado com estudantes universitários, a partir de uma amostra de conveniência, composta por 202 estudantes sendo 83,7\% $(n=169)$ do curso de Informática de universidade privada e 16,3\% ( $\mathrm{n}=33$ ) do curso de Psicologia de universidade pública, com predomínio de estudantes do sexo masculino $(75,7 \%)(\mathrm{n}=169)$ e com idade variando entre 16 e 49 anos, com média de 22,84 ( $D P=5,92)$. Em relação ao período do curso, predominaram alunos de primeiro período $(86,7 \%)$, daí possivelmente o percentual tão baixo de reprovação durante a graduação.

\section{Instrumentos}

O questionário aplicado continha quatro seções. A primeira era referente à caracterização da amostra e continha aos dados sociodemográficos para coletar informações como idade, sexo, instituição, classe social e período do curso. As três outras seções do questionário destinavam-se às três escalas que foram aplicadas e que são descritas a seguir.
O Inventário de Habilidades Sociais (IHS-DelPrette) (Del Prette \& Del Prette, 2001) é um instrumento de autorrelato para avaliação de habilidades sociais, analisado e aprovado pelo Conselho Federal de Psicologia e disponibilizado em forma de uma caixa com materiais impressos (manual, fichas de aplicação e de apuração), com opção de apuração informatizada on-line. Compõe-se de 38 itens, cada um descrevendo uma situação de relação interpessoal e uma demanda de habilidade para reagir àquela situação. O respondente deve estimar a frequência com que reage da forma sugerida em cada item, considerando o total de vezes que se encontrou na situação descrita, e estimar a frequência de sua resposta em escala tipo Likert, com cinco pontos, variando de zero (nunca ou raramente) a 4 (sempre ou quase sempre). Em alguns itens há um fraseado "negativo", ou seja, pontuação mais alta indica déficit de habilidades sociais, devendo-se, nestes, inverter a pontuação para a obtenção do escore. O IHS-Del-Prette produz um escore geral, referenciado à norma em termos de percentis, e escores em cinco subescalas de habilidades sociais: F1 Enfrentamento e Autoafirmação com Risco; F2 - Autoafirmação na Expressão de Sentimento Positivo; F3 - Conversação e Desenvoltura Social, F4 - Autoexposição a Desconhecidos e Situações Novas, e F5 - Autocontrole da Agressividade. Para responder, os participantes seguem uma escala tipo Likert, com cinco pontos variando de 0 (nunca ou raramente) e 4 (sempre ou quase sempre). O estudo de Del Prette et al. (1998) identificou qualidades psicométricas adequadas e o inventário foi aprovado pelo Conselho Federal de Psicologia.

Outro estudo realizado por Del Prette, Del Prette e Barreto (1999) constatou a sensibilidade do IHS para detectar modificações produzidas em um grupo de alunos universitários, com aumento das habilidades sociais no grupo treinado e ausência de efeito no grupo controle. Este último serviu também para atestar a estabilidade temporal do IHS, uma vez que foi feito teste e re-teste no grupo controle. É importante destacar também o estudo realizado por Bandeira et al. (2000) em que estudantes de Psicologia responderam ao IHS e à Escala de Assertividade de Rathus, revelando correlação significativa entre as duas escalas. Na aplicação teste-reteste do IHS foi encontrada correlação significativa entre as duas aplicações, confirmando a estabilidade temporal do instrumento e a sua adequação para a aplicação em universitários no Brasil. 
A construção e validação da Escala de Avaliação da Vida Acadêmica (EAVA) foi realizada por Vendramini et al. (2004) com o objetivo de captar a autopercepção do estudante sobre sua vivência universitária. O estudo foi feito com uma amostra de 1118 universitários, com predominância do sexo masculino (54\%) e idades variando de 19 a 62 anos. O indivíduo, ao responder ao instrumento, deve avaliar o quanto concorda ou discorda com os comportamentos acadêmicos descritos no item, utilizando uma escala tipo Likert, que varia de 1 (discordo totalmente) a 5 (concordo totalmente). Os autores extraíram do instrumento cinco fatores, quais sejam: (a) ambiente universitário ("Estou satisfeito(a) com as atividades culturais propostas pela Universidade"), (b) compromisso com o curso ("Tenho certeza que escolhi o curso certo"), (c) habilidade do estudante ("O meu curso não está correspondendo às minhas aptidões e habilidades"), (d) envolvimento em atividades não-obrigatórias ("Participo das atividades culturais e artísticas promovidas pela Universidade") e (e) condições para o estudo ("Não tenho tempo para realizar as atividades extraclasse"), com $43 \%$ de variância total explicada e coeficientes de fidedignidade variando de 0,63 a 0,80 . A versão final da escala foi de 34 itens e com um alpha de Cronbach de 0,87 satisfatório, sinalizando que o instrumento apresenta índices psicométricos adequados, podendo ser utilizados em estudantes universitários brasileiros para a avaliação dos fatores da vivência acadêmica e desse construto como um todo.

A versão reduzida do Questionário de Vivência Acadêmica (QVA-r) foi elaborada em Portugal por Almeida, Soares e Ferreira (2002) com o objetivo de ter uma medida que pudesse avaliar as vivências acadêmicas sem o uso de um questionário muito longo. De acordo com Santos et al. (2006), estudos têm mostrado boas qualidades psicométricas da versão reduzida. O QVA-r tem um total de 60 itens e reúne cinco dimensões da adaptação acadêmica, a saber: (a) pessoal, (b) interpessoal, (c) institucional, (d) carreira e (e) estudo. O estudante deve expressar sua opinião, se de acordo ou em desacordo, em função da adequação da situação proposta com o seu sentimento naquele momento sobre suas vivências acadêmicas. O indivíduo, ao responder ao instrumento, deve avaliar o quanto concorda ou discorda com os comportamentos acadêmicos descritos no item, utilizando uma escala tipo Likert, que varia de 1 (Nada a ver comigo, totalmente em desacordo, nunca acontece) a 5 (Tudo a ver comigo, totalmente de acordo, acontece sempre). São exemplos de itens deste instrumento: "Faço amigos com facilidade na minha Universidade/Faculdade" e "Sou pontual na chegada às aulas".

A análise fatorial da versão brasileira permitiu a identificação dos mesmos cinco fatores da escala original, explicando $40 \%$ da variância, porém com a exclusão de cinco itens, ficando a versão final adaptada ao Brasil com 55 itens. A análise de consistência interna de cada uma das cinco dimensões foi satisfatória com coeficientes de fidedignidade variando entre 0,86 (carreira) e 0,77 (estudo) e da escala total equivalente a 0,88 .

\section{Procedimentos de coleta de dados}

Após a autorização do Comitê de Ética (processo $\mathrm{n}^{\mathrm{o}}$ 129/2009 aprovado em 25/02/2010), deu-se início à coleta de dados com os estudantes voluntários que assinaram o Termo de Consentimento Livre e Esclarecido e preencheram um questionário de dados sociodemográficos. A aplicação do instrumento foi coletiva, em sala de aula, com respostas individuais em tempo livre e esclarecimento de que os dados seriam tratados coletivamente, com preservação do sigilo das respostas individuais. Todos os instrumentos foram aplicados em uma única sessão.

\section{Procedimentos de análise de dados}

Em relação à análise de dados, os procedimentos seguiram três etapas. Na primeira foram calculados os coeficientes de fidedignidade de cada fator das três escalas e criadas, no banco de dados, as variáveis correspondentes a cada fator e aos escores totais de cada uma das três escalas. Em uma segunda etapa, foram realizados os procedimentos de estatística descritiva, sendo calculados os valores de média e desvio-padrão das respostas relativas a cada dimensão e ao escore total das três escalas. Nesta etapa foram ainda examinadas as distribuições univariadas e bivariadas por meio de métodos gráficos (diagrama de ramo e folha, boxplot) e numéricos (medidas de simetria) com o objetivo de avaliar normalidade, homogeneidade de variância e a presença de dados extremos (outliers, 3 desvios-padrões acima ou abaixo da média). Na terceira e última etapa foram realizadas as análises inferenciais. Assim, foram realizadas correlações de Pearson e teste $t$ de Student para amostras independentes. As correlações compreenderam análises bivariadas 
entre os escores totais e os fatores dos três instrumentos, permitindo a análise intra-testes e inter-testes. $\mathrm{O}$ teste $t$, por sua vez, permitiu comparar as médias por curso e por gênero.

\section{RESULTADOS}

Este estudo teve como objetivos caracterizar os universitários com relação ao seu repertório de habilidades sociais e à vivência acadêmica, examinar a cor- relação entre tais variáveis e comparar diferenças por gênero e curso. Tendo em vista que o objetivo do estudo de caracterizar os universitários com relação ao seu repertório de habilidades sociais e à vivência acadêmica, é apresentada a Tabela 1 , que descreve as médias e os desvios-padrão de cada fator das escalas, além de apresentar também os valores dos coeficientes de fidedignidade, medidos pelo Alpha de Cronbach.

Tabela 1

Coeficiente de fidedignidade, médias e desvios-padrão de cada fator das escalas

\begin{tabular}{clccc} 
Escala & Fatores & Alpha de Cronbach & Média & Desvio-padrão \\
\hline \multirow{2}{*}{ IHS } & Assertividade & 0,76 & 3,29 & 0,68 \\
& Autoafirmação & 0,70 & 3,82 & 0,71 \\
& Conversação e desenvoltura social & 0,57 & 3,78 & 0,62 \\
& Autoexposição a desconhecidos e situações novas & 0,61 & 3,31 & 0,90 \\
& Autocontrole da agressividade a situações adversas & 0,34 & 3,81 & 0,78 \\
& IHS ESCORE TOTAL & 0,85 & 3,59 & 0,47 \\
& Carreira & 0,86 & 4,18 & 0,50 \\
& Estudo & 0,77 & 3,44 & 0,58 \\
QVA-r & Institucional & 0,58 & 3,63 & 0,47 \\
& Pessoal & 0,58 & 0,65 \\
& Interpessoal & 0,86 & 3,51 & 0,61 \\
& QVA-r ESCORE TOTAL & 0,85 & 3,67 & 0,40 \\
& Ambiente universitário & 0,91 & 3,70 & 0,45 \\
& Compromisso com o curso & 0,50 & 3,59 & 0,62 \\
EAVA & Habilidade do estudante & 0,75 & 4,21 & 0,51 \\
\cline { 2 - 4 } & Envolvimento em atividades não-obrigatórias & 0,73 & 3,56 & 0,78 \\
Condições para o estudo e desempenho acadêmico & 0,76 & 2,94 & 0,72 \\
EAVA ESCORE TOTAL & 0,41 & 3,25 & 0,39 \\
\hline
\end{tabular}

No que diz respeito aos escores das três escalas, a amplitude de variação foi de 5 pontos, sendo que a escala IHS varia de zero a quatro e as demais de um a cinco. Para facilitar a comparação entre essas escalas, considerou-se para o IHS a amplitude de variação de 1 a 5, como nas demais escalas. Os resultados apontaram, portanto, para escores ligeiramente mais altos para escala QVA-r do que para as escalas EAVA e IHS, cujos escores foram muito próximos, com um pouco mais de variabilidade nas respostas do IHS. Nos resultados das três medidas observa-se certa homogeneidade nas respostas dos pesquisados, uma vez que os coeficientes de variação foram de $13,1 \%$ (IHS); 10,8\% (QVA-r) e 10,9\% (EAVA).

Analisando-se os resultados específicos de cada escala, observa-se por um lado que os fatores com médias mais elevadas no IHS foram Autoafirmação, Autocontrole da agressividade a situações adversas e conversação e desenvoltura social. Por outro lado, os fatores com escores mais baixos foram Assertividade e Autoexposição a desconhecidos e situações novas. Em relação à escala QVA-r, o escore mais alto foi Carreira e o mais baixo Estudo. Por fim, no EAVA, o fator com média mais alta foi Compromisso com o 
curso e o fator com média mais baixa foi Envolvimento em atividades não obrigatórias.

Com o objetivo de explorar a relação entre os construtos, foram realizadas correlações de Pearson, que são apresentadas na Tabela 2. Tais correlações contemplaram tanto os escores totais das três escalas, como também os fatores que compõem cada uma delas.

Os resultados apresentados na Tabela 2 apontaram para um conjunto de correlações significativas entre os fatores das três escalas e entre as escalas entre si. Das 153 correlações bivariadas possíveis, $65 \%$ foram significativas. Ao analisar a magnitude dos coeficientes de correlação, segundo a classificação de Miles e Shevlin (2001), que agrupa as correlações em três níveis: até 0,29 (baixa); 0,30 a 0,49 (moderada); acima de 0,50 (elevada), percebe-se um predomínio das correlações baixas (46\%), seguidas pelas correlações moderadas $(35 \%)$, sendo as menos frequentes as correlações elevadas $(19 \%)$.

Quando se separa a análise das correlações intratestes e inter-testes, observa-se que, como era de se esperar, a maior concentração de correlações elevadas concentra-se entre as dimensões de cada uma das escalas e o escore total dessa escala (intra-teste). $\mathrm{Na}$ verdade, das 19 correlações elevadas encontradas, 16 são do escore total da escala com suas respectivas dimensões. As outras três correlações elevadas diziam respeito às correlações entre: (a) as dimensões Carreira e Estudo da Escala de QVA-r; (b) as dimensões Assertividade e Autoafirmação da Escala IHS; e (c) a dimensão Carreira da Escala QVA-r e a dimensão Compromisso com o curso da Escala EAVA.

As demais correlações foram classificadas de baixas a moderadas, o que sinaliza grau relativamente baixo de variância comum entre os fatores das escalas de integração acadêmica e os fatores do inventário de habilidades sociais. Ainda sobre as correlações intratestes, vale destacar que houve correlações significativas de todas as dimensões de cada escala com as demais dimensões da mesma escala, sendo dois terços dessas correlações de magnitude moderadas/elevadas e apenas um terço baixas. Merecem destaque as correlações entre as dimensões do QVA-r, com apenas $20 \%$ das correlações internas classificadas como baixas.

No que diz respeito às correlações entretestes, as escalas QVA-r e EAVA apresentaram correlação de magnitude elevada, segundo a classificação de Miles e Shevlin (2001), o que seria de se esperar já que as duas têm o mesmo propósito de avaliar as vivências acadêmicas. Seguindo a classificação proposta por tais autores, a correlação das duas com o IHS, embora significativas, tiveram baixa magnitude.

Além desses resultados de correlação entre os construtos de vivência acadêmica e habilidades sociais, atendendo-se ao objetivo da pesquisa, realizou-se também um estudo comparativo das médias por gênero e por curso. A Tabela 3 apresenta os resultados dos escores totais e cada um dos fatores que compõem as três escalas pesquisadas, considerando-se as médias que foram significativamente diferentes de acordo com o gênero. 
218 Adriana Benevides Soares; Luciana Mourão; Acácia Aparecida Angeli dos Santos \& Thatiana Valory dos Santos Mello

Tabela 2

Correlações de Pearson entre as escalas EAVA, QVA-r e IHS e seus fatores

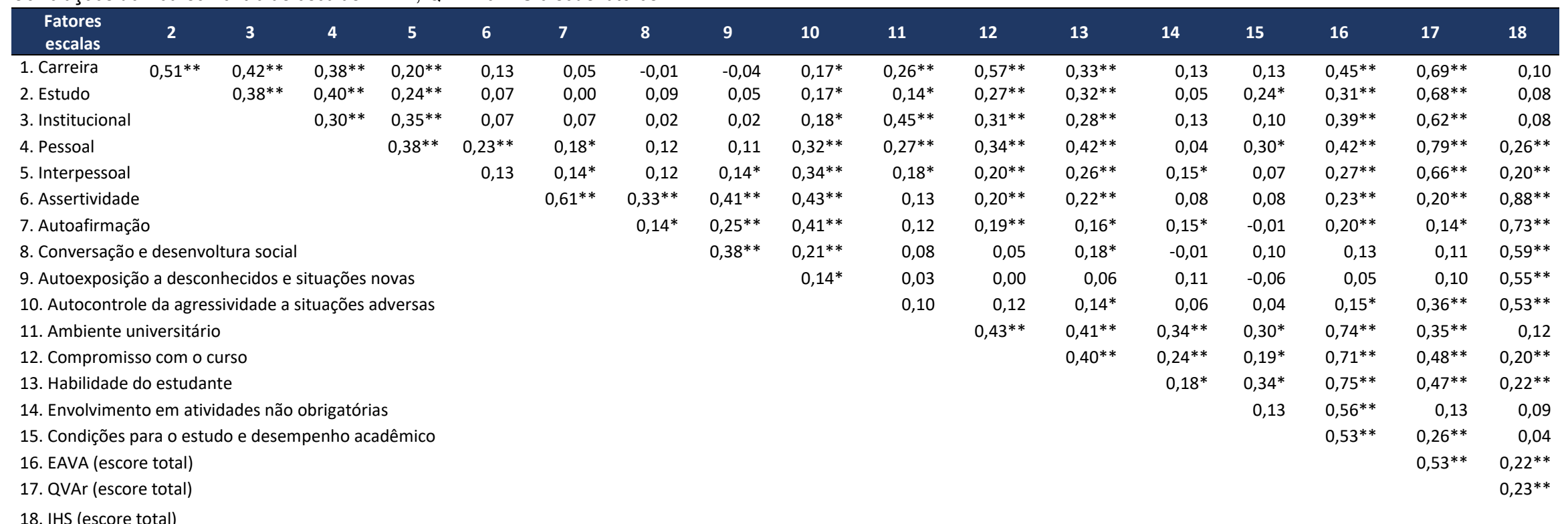

Nota. ${ }^{*} \mathrm{p}<0,05 ; * * \mathrm{p}<0,01$.

Tabela 3

Teste t para comparação de médias de acordo com o gênero

\begin{tabular}{|c|c|c|c|c|c|c|}
\hline Escala & Fator & Gênero & $\mathbf{N}$ & Média & Desvio-padrão & Teste $t$ \\
\hline \multirow[t]{8}{*}{ QVAr } & Carreira & Masculino & 146 & 4,23 & 0,47 & $t(191)=2,22^{*}$ \\
\hline & & Feminino & 47 & 4,05 & 0,55 & \\
\hline & Institucional & Masculino & 146 & 3,67 & 0,49 & $t(191)=2,06^{*}$ \\
\hline & & Feminino & 47 & 3,51 & 0,45 & \\
\hline & Pessoal & Masculino & 146 & 3,61 & 0,61 & $t(191)=3,51 * *$ \\
\hline & & Feminino & 47 & 3,24 & 0,68 & \\
\hline & QVAr Escore total & Masculino & 146 & 3,74 & 0,41 & $t(191)=2,67^{* *}$ \\
\hline & & Feminino & 47 & 3,58 & 0,37 & \\
\hline \multirow[t]{2}{*}{ IHS } & Autocontrole da agressividade & Masculino & 146 & 2,91 & 0,79 & $t(191)=2,37^{*}$ \\
\hline & & Feminino & 47 & 2,57 & 0,70 & \\
\hline
\end{tabular}

Nota. ${ }^{*} p<0,05 ; * * p<0,01$. 
Os resultados dos testes $t$ para amostras independentes mostraram que, há diferenças significativas entre estudantes do sexo masculino e feminino nas médias de três dimensões de QVAr (Carreira, Institucional e Pessoal), além do próprio escore total da escala. Em todos os fatores, homens apresentaram médias mais elevadas do que mulheres. O mesmo também aconteceu em relação à dimensão autocontrole da agressividade a situações adversas. As demais dimen- sões do QVAr e do IHS, assim como todas as dimensões da EAVA, não apresentaram diferenças de médias significativas em relação ao gênero.

Por fim, foi realizada a análise comparativa, por curso, das médias dos fatores e dos escores totais das três escalas. A Tabela 4 apresenta os resultados do teste $t$ com os valores das médias relativas aos estudantes de Psicologia e de Informática.

\section{Tabela 4}

Teste t para comparação de médias de acordo com o curso superior

\begin{tabular}{|c|c|c|c|c|c|c|}
\hline Escala & Fator & Curso & $\mathbf{N}$ & Média & Desvio-padrão & Teste $t$ \\
\hline \multirow[t]{4}{*}{ QVAr } & \multirow{2}{*}{ Pessoal } & Inform. & 169 & 3,59 & 0,61 & \multirow{2}{*}{$t(200)=4,40 * *$} \\
\hline & & Psicol. & 33 & 3,07 & 0,67 & \\
\hline & \multirow{2}{*}{$\begin{array}{l}\text { QVAr } \\
\text { Escore total }\end{array}$} & Inform. & 169 & 3,73 & 0,38 & \multirow{2}{*}{$t(200)=2,72 * *$} \\
\hline & & Psicol. & 33 & 3,53 & 0,44 & \\
\hline \multirow[t]{6}{*}{ IHS } & \multirow{2}{*}{ Autoafirmação } & Inform. & 169 & 2,77 & 0,74 & \multirow{2}{*}{$t(200)=-2,36^{*}$} \\
\hline & & Psicol. & 33 & 3,08 & 0,46 & \\
\hline & \multirow{2}{*}{$\begin{array}{l}\text { Autoexposição a desconhecidos } \\
\text { e situações novas }\end{array}$} & Inform. & 169 & 2,25 & 0,89 & \multirow{2}{*}{$t(200)=-2,34^{*}$} \\
\hline & & Psicol. & 33 & 2,64 & 0,92 & \\
\hline & \multirow{2}{*}{ Autocontrole da agressividade } & Inform. & 169 & 2,87 & 0,79 & \multirow{2}{*}{$t(200)=2,47^{*}$} \\
\hline & & Psicol. & 33 & 2,51 & 0,69 & \\
\hline \multirow[t]{10}{*}{ EAVA } & \multirow{2}{*}{ Compromisso com o curso } & Inform. & 169 & 4,25 & 0,57 & \multirow{2}{*}{$t(200)=2,00 *$} \\
\hline & & Psicol. & 33 & 4,02 & 0,78 & \\
\hline & \multirow{2}{*}{ Habilidade do estudante } & Inform. & 169 & 3,61 & 0,51 & \multirow{2}{*}{$t(200)=2,85^{* *}$} \\
\hline & & Psicol. & 33 & 3,34 & 0,47 & \\
\hline & \multirow{2}{*}{$\begin{array}{l}\text { Envolvimento em atividades } \\
\text { não obrigatórias }\end{array}$} & Inform. & 169 & 2,87 & 0,78 & \multirow{2}{*}{$t(200)=-2,82^{* *}$} \\
\hline & & Psicol. & 33 & 3,28 & 0,69 & \\
\hline & \multirow{2}{*}{$\begin{array}{l}\text { Condições p/estudo e } \\
\text { desempenho acadêmico }\end{array}$} & Inform. & 169 & 3,35 & 0,67 & \multirow{2}{*}{$t(200)=4,99 * *$} \\
\hline & & Psicol. & 33 & 2,71 & 0,74 & \\
\hline & EAVA & Inform. & 169 & 3,60 & 0,38 & \multirow{2}{*}{$t(200)=2,23^{*}$} \\
\hline & Escore total & Psicol. & 33 & 3,44 & 0,40 & \\
\hline
\end{tabular}

Nota. ${ }^{*} \mathrm{p}<0,05 ;{ }^{* *} \mathrm{p}<0,01$.

De acordo com o resultado do teste $t$, houve diferenças de acordo com o curso superior. O escore total da EAVA e todas as suas dimensões (exceto ambiente universitário) apresentaram diferenças significativas nas médias. Em todos os casos, as médias dos alunos de Informática foram mais elevadas do que as dos estudantes de Psicologia, exceto para a dimensão Envolvimento em atividades não-obrigatórias, que teve média mais alta nos alunos de Psicologia. Já em rela- ção às habilidades sociais, os respondentes do curso de Informática tenderam a apresentar médias de $\mathrm{Au}$ toafirmação e de Autoexposição a desconhecidos e a situações novas mais baixos que os respondentes do curso de Psicologia. Porém, esses últimos apresentam menor Autocontrole da agressividade a situações adversas do que os alunos de Informática. Em relação às dimensões da QVAr, apenas a dimensão Pessoal apresentou diferença significativa, sendo que os estudantes 
de Psicologia apresentaram escores mais elevados que os estudantes de Informática

\section{DISCUSSÃO}

Os resultados apontaram, portanto, para escores ligeiramente mais altos para escala QVA-r do que para as escalas EAVA e IHS. Este resultado possivelmente esteja relacionado a atenção crescente que vem sendo dedicada à temática da adaptação à vida acadêmica e combate à evasão (Igue, Bariani, \& Milanesi, 2008; Santos, Suehiro, Oliveira, Cunha, \& Carrilho, 2006; Soares, Poubel, \& Mello, 2009). O fato de na Escala de Avaliação da Vida Acadêmica ter sido mais baixa a média de Envolvimento em atividades nãoobrigatórias não era esperado, uma vez que a amostra é predominantemente masculina e que no estudo de Ferreira, Almeida e Soares (2001) o envolvimento em atividades extracurriculares era mais comum entre os homens.

No que concerne aos resultados nas correlações inter e entre testes, estudos como os de Gerk e Cunha (2006), Soares et al. (2009) e Baldez (2009) identificaram diversas correlações positivas entre os fatores de habilidades sociais e de adaptação acadêmica, indicando que elas podem facilitar a aderência à universidade e diminuir a evasão escolar. Embora a competência social esteja relacionada à capacidade favorável de se adaptar a situações sociais novas e interagir com outras pessoas (Del Prette et al., 2012; Mota, Matos, $\&$ Lemos, 2011), sendo considerada um fator de proteção ao indivíduo no enfrentamento de situações estressantes que podem resultar do ingresso em um novo ambiente, no caso a universidade (Del Prette \& Del Prette, 2009), as correlações encontradas no presente estudo foram em grande parte fracas ou ainda inexistentes.

Os estudos de Baker e Siryk, (1989), Tinto (1975), Terenzini, Lorang e Pascarella (1981) e Pascarela e Terenzini (2005) atribuíram importância aos elementos interpessoais e relacionais no modelo de ajustamento acadêmico assim como Soares et al. (2006) verificaram que autonomia emocional impactava na vida acadêmica e, desta forma, os resultados encontrados são diferentes do que era possível supor. Além disso, vários estudos têm apontado fortes associações entre as habilidades sociais e a competência social para melhores vivências acadêmicas e, consequentemente, melhor adaptação ao Ensino Superior (Baldez;
2009; Gerk \& Cunha, 2006; Soares, Poubel, \& Melo, 2009; Soares et al. 2013).

No que diz respeito aos resultados quanto ao gênero e curso, o fato de a amostra do presente estudo ter tido um número maior de respondentes do sexo masculino e alunos do curso de graduação em computação é um aspecto que merece ser analisado com atenção. Estudos anteriores que usaram os mesmos instrumentos mostram que, em relação ao gênero, não há diferenças significativas no estudo original de validação da EAVA (Vendramini et al., 2004), e tampouco no estudo original português do QVA-r (Almeida, Soares, \& Ferreira, 2002) em que haviam em sua maioria mulheres. Contudo o trabalho de Granado et al. (2005) apontou que os estudantes do sexo feminino apresentam resultados significativamente mais favoráveis nas dimensões Carreira, Interpessoal e Estudo do QVA-r, assim como em seu escore geral. Também o estudo de Soares et al. (2013) em que a amostra era predominantemente constituída de participantes do gênero feminino identificou resultados semelhantes com mulheres tendo melhor desempenho na dimensão interpessoal e estudo. Os resultados de comparação por gênero podem ser úteis não apenas para que se conheçam as diferenças de padrão de vivência acadêmica e de repertório de habilidades sociais, mas também para que possam ser pensadas ações de integração acadêmica que visem desenvolver a vivência acadêmica e as habilidades sociais dos grupos com menores escores nessas variáveis.

No que concerne ao IHS (Del Prette \& Del Prette, 2001), diferenças significativas têm sido apontadas entre estudantes universitários (Bueno, Oliveira, \& Oliveira, 2001; Echavarri, Godoy, \& Olaz, 2007). No caso dos estudantes universitários verifica-se médias mais elevadas para habilidades de Enfrentamento com risco para os homens e Autoafirmação na expressão de afetos positivos para as mulheres (Bueno, Oliveira, \& Oliveira, 2001; Soares et al. 2013), reafirmando um capital cultural reforçado historicamente de que homens são mais valorizados por sua assertividade e as mulheres por sua afetividade. Corroboram estes resultados também o estudo de Soares et al. (2013) com estudantes universitários em que foram encontrados resultados superiores para os participantes do gênero masculino no que diz respeito as habilidades sociais de enfrentamento e autoafirmação com risco.

Alguns outros estudos, como os de Costa e Campos (1986) e Ferreira et al. (2001), apontam para dife- 
renças de gênero entre a identidade de estudantes universitários, a adaptação acadêmica e o envolvimento extracurricular. Oyarzun Iturra, Estrada Goic, Pino Astete e Oyarzun Jara (2012) também apontam para diferenças de gênero associadas a habilidades sociais e rendimento acadêmico. As moças parecem aproveitar mais que os rapazes a qualidade de seus relacionamentos pessoais tais como cooperação e colaboração para melhorar seu desempenho acadêmico. Assim, a despeito da contribuição para a literatura da área que provêm deste estudo, seria importante que novos estudos fossem realizados com diferentes amostras ou com maior equilíbrio entre gênero e curso de forma a permitir mais discussões acerca destas variáveis sobre os construtos Vivência Acadêmica, Avaliação da Vida Acadêmica e Habilidades Sociais.

\section{CONSIDERAÇÕES FINAIS}

$\mathrm{O}$ estudo dos construtos habilidades sociais e vivências acadêmicas permitem que seus resultados possam subsidiar políticas e práticas universitárias tendo em vista a promoção da adaptação e do sucesso acadêmico sem que esta prática esteja circunscrita no desempenho escolar. Alguns resultados da presente pesquisa que podem ser considerados na práxis da área são as correlações inter e entre testes do QVAr, EAVA e IHS que indicam diferenças entre os instrumentos, com correlações predominantemente de baixa magnitude entre eles. Também se conclui que há diferenças significativas entre os escores médios do QVAr, EAVA e IHS, tanto por gênero quanto por curso, o que pode ensejar políticas específicas por parte das universidades para seus diferentes públicos de estudantes.

É importante trazer ao final do estudo, algumas limitações identificadas, especialmente voltadas para as características peculiares da amostra. Tais características e o fato de a amostra ter sido acidental impedem que os resultados possam ser extrapolados para outros contextos. Outrossim, vale ressaltar que a escolha dos construtos não pretendeu dar conta de toda a gama de experiências relacionadas à vida universitária, não se permitindo que sejam dadas todas as explicações que se fazem necessárias.

Acredita-se que o presente estudo pôde fornecer novos elementos para a compreensão das vivências acadêmicas e das habilidades sociais de estudantes universitários no Brasil, contribuindo para a amplia- ção do debate e de novas proposições. Por fim, destaca-se a relevância da realização de novos estudos sobre o tema, considerando que o ensino superior deve promover o desenvolvimento global do aluno (competências, controle das emoções, desenvolvimento de autonomia, das relações interpessoais, da identidade, dos projetos pessoais, dos ideais e da integridade) do jovem adulto e não apenas proporcionar a transmissão de conhecimentos e o desenvolvimento intelectual.

\section{REFERÊNCIAS}

Almeida, L. S., \& Soares, A. P. (2003). Os estudantes universitários: sucesso escolar e desenvolvimento psicossocial. In E. Mercuri \& S. A. J. Polydoro (Orgs.), Estudante universitário: características e experiências de formação (pp. 15-40). Taubaté: Cabral.

Almeida, L. S., Soares, A. P. C., \& Ferreira, J. A. (2002). Questionário de Vivências Acadêmicas (QVA-r): avaliação do ajustamento dos estudantes universitários. Avaliação Psicológica, 1(2), 81-93.

Almeida, L. S., Soares, A. P. C., \& Ferreira, J. A. G. (1999). Adaptação e rendimento acadêmico no ensino superior: fundamentação e validação de uma escala de vivências acadêmicas. Psicologia: Teoria, Investigação e Prática, 1, 157-170.

Almeida, L., Marinho-Araujo, C. M., Amaral, A., \& Dias, D. (2012). Democratização do acesso e do sucesso no ensino superior: uma reflexão a partir das realidades de Portugal e do Brasil. Avaliação (Campinas), 17, 899920.

Azevedo, A., \& Faria, L. (2001). Impacto das condições pessoais e contextuais na transição do ensino secundário para o ensino superior. Revista da UFP, 6, 257-269.

Baker, R. W., \& Siryk, B. S. (1989). SACQ Student adaptation to college questionnaire: Manual. Los Angeles, CA: Western Psychological Services, WPS.

Baldez, M. (2009). A relação entre as habilidades sociais e a adaptação dos estudantes ao ensino superior: um estudo no estado do Rio de Janeiro. Dissertação em Psicologia. Universidade Salgado de Oliveira.

Bandeira, M., \& Quaglia, M. A. C. (2005). Habilidades sociais de estudantes universitários: Identificação de situações sociais significativas. Interação em Psicologia, 9(1), 45-55.

Bandeira, M., Costa, M. N., Del Prette, Z. A. P., Del Prette, A., \& Gerk-Carneiro, E. (2000). Qualidades psicométricas do Inventário de Habilidades Sociais (IHS): estudo sobre a estabilidade temporal e a validade concomitante. Estudos de Psicologia (Natal), 5, 401-419. 
Bandura, M., Silva, S., Cordeiro, L., Pereira, Z. A. P., \& Del Prette, A. (2006) Habilidades sociais e variáveis sociodemográficas em estudantes do ensino fundamental. Psicología Psicologia em Estudo, 11(3), 541-549.

Barreto, M. C. M., Pierre, M. R. S. R., Del Prette, Z. A. P., \& Del Prette, A. (2004). Habilidades Sociais entre Jovens Universitários: um estudo comparativo. Revista de Matemática e Estatística, 22(1), 31-42.

Bartholomeu, D., Nunes, C. H. S. S., \& Machado, A. A. (2008) Traços de personalidade e habilidades sociais em universitários. Psico-USF, 13, 41-50.

Bolsoni-Silva, A. T., Loureiro, S. R., Rosa, C. F., \& de Oliveira, M. C. F. A. (2010). Caracterização das habilidades sociais de universitários. Contextos Clínicos, 3(1), 62-75.

Bueno, J. M. H., Oliveira, S. M. S. S., \& Oliveira, J. C. S. (2001). Um estudo correlacional entre habilidades sociais e traços de personalidade. Psico-USF, 6, 1, 31-38.

Costa, M. \& Campos, B. P. (1986). Identidade de estudantes universitários: diferenças de curso e sexo. Cadernos e Consulta Psicológica, 2, 5-11.

Cunha, S. M. \& Carrilho, D. M. (2005) O processo de adaptação ao ensino superior e o rendimento acadêmico, Psicologia Escolar e Educacional, 9, 215-224.

Del Prette, A., \& Del Prette, Z. A. P. (2001). Inventário de Habilidades Sociais - IHS. Editora Casa do Psicólogo.

Del Prette, A., \& Del Prette, Z. A. P. (2009). Adolescência e Fatores de Risco: Habilidades Sociais e Fatores de Risco: A importância das habilidades sociais educativas. In Haase, V. G., Ferreira, F. O. \& Penna, F. J. (Orgs.), Aspectos biopsicossociais da saúde na infância e adolescência (pp. 503-522). Belo Horizonte: Coopmed.

Del Prette, A., Del Prette, Z. A. P., \& Barreto, M. C. M. (1999). Habilidades sociales en la formación del psicólogo: Análisis de un programa de intervención. Psicología Conductual, 7, 27-47.

Del Prette, Z. A. P. \& Del Prette, A. (1983). Análise do repertório assertivo em estudantes de Psicologia. Revista de Psicologia, 1(1), 15-24.

Del Prette, Z. A. P., Del Prette, A., \& Barreto, M. C. M. (1998). Análise de um Inventário de Habilidades Sociais (IHS) em uma amostra de universitários. Psicologia: Teoria e Pesquisa, 14, 219-228.

Del Prette, Z. A. P., Del Prette, A., Oliveira, L. A., Gresham, F. M., \& Vance, M. J. (2012). Role of social performance in predicting learning problems: Prediction of risk using logisticregression analysis. School Psychology International, Journal, 2, 1-16.

Echavarri, M., Carlos Godoy, J., \& Olaz, F. (2007). Diferencias de género en habilidades cognitivas y rendimien- to académico en estudiantes universitarios. Universitas Psychologica, 6, 2, 319-329.

Ferreira, J. A., Almeida, L. S., \& Soares, A. P. C. (2001). Adaptação acadêmica em estudante do $1^{\circ}$ ano: diferenças de gênero, situação do estudante e curso. Psico-USF, 6 , $1-10$.

Gerk, E., \& Cunha, S. (2006). Habilidades sociais na adaptação de estudantes ao ensino superior. In M. Bandeira, A. Prette \& Z. Del Prette (Orgs.), Estudos sobre habilidades sociais e relacionamento interpessoal (pp. 181198). São Paulo: Casa do Psicólogo.

Granado, J. I. F., Santos, A. A. A., Almeida, L. S., Soares, A. P., \& Guisande, M. A. (2005). Integração académica de estudantes universitários: contributos para a adaptação e validação do QVA-r no Brasil. Psicologia e Educação, 12(2), 31-43.

Hair J. R., Black, W. C., Babin, B. J., Anderson, R. E., \& Tatham, R. L. (2009). Análise multivariada de dados. $6^{\text {a }}$ ed. Porto Alegre: Bookman.

Igue, E., Bariani, I., \& Milanesi, P. (2008). Vivência acadêmica e expectativas de universitários ingressantes e concluintes. Psico-USF, 13, 155-164.

Miles, J., \& Shevlin, M. (2001) Applying regression \& correlation. A guide for students and researches. London: SAGE Publications.

Mota, C. P., Matos, P. M., \& Lemos, M. S. (2011). Psychometric properties of the social skills questionnaire: Portuguese adaptation of the student form (Grades 7 to 12). The Spanish Journal of Psychology, 14(1), 486-499.

Oyarzun Iturra, G., Estrada Goic, C., Pino Astete, E., \& Oyarzun Jara, M. (2012) Habilidades sociales y rendimiento académico: una mirada desde el género. Acta Colombiana de Psicologia, 15, 2, 21-28.

Pagotti, G., \& Pagotti, A. (2005). As preocupações dos estudantes universitários: um estudo exploratório. Revista Psicopedagogia, 22(68), 94-104.

Pires, H. S., Almeida, L., \& Ferreira, J. A. (2000). Questionário de Vivências Acadêmicas (QVA) aos estudantes universitários dos PALOP, Em In A. P. Soares, A. Osório. J. V. Capela, L. S. Almeida, R. M. Vasconcelos \& S. M. Caíres (Orgs.). Transição para o Ensino Superior. Braga, Portugal: Universidade do Minho.

Polydoro, S. A. J., Primi, R., Serpa, M. N. F., Zaroni, M. M. H., \& Pombal, K. C. P. (2001). Desenvolvimento de uma escala de integração ao ensino superior. Psico-USF, $6,11-18$.

Santos, A. A. A., Suehiro, A. C. B., Oliveira, E. Z., Cunha, S. M., \& Carrilho, D. M. (2006). Avaliação da integração acadêmica no ensino superior: estudo com estudantes de engenharia. Psicologia e Educação, 13(2), 79-89. 
Soares, A. B., Francischetto, V., Peçanha, A. P. C. L. P., Miranda, J. M., \& Dutra, B. M. S. (2013). Inteligência e competência social na adaptação à universidade, Estudos de Psicologia (Campinas), 30(3), 317-328.

Soares, A. B., Poubel, L. N., \& Mello, T. V. S. (2009). Habilidades sociais e adaptação acadêmica: um estudo comparativo em instituições de ensino público e privado. Aletheia, 30(2), 27-42.

Soares, A. P., Almeida, L. S., Diniz, A. M., Guisande, M. A. (2006). Modelo Multidimensional de Ajustamento de jovens ao contexto Universitário (MMAU): Estudo com estudantes de ciências e tecnologias versus ciências sociais e humanas. Análise Psicológica, 1 (XXIV): 15-27.

Teixeira, M. A. P., Castro, G. D., \& Piccolo, L. R. (2007). Adaptação à Universidade em estudantes Universitários: Um Estudo Correlacional. Interação em Psicologia, 11, 211-220.

Teixeira, M. A. P., Dias, A. C. G., Wottrich, S. H., \& Oliveira, A. M. (2008). Adaptação à universidade em jovens calouros. Psicologia Escolar e Educacional, 12(1), 185202.
Terenzini, P. T., Lorang, W. G., \& Pascarella, E. T. (1981). Predicting freshman persistence and voluntary dropout decisions: A replication. Research in Higher Education, $15,109-127$.

Tinto, V. (1975). Dropout form higher education - Theoretical synthesis of recent research. Review of Educational Research, 45, 89-125.

Vendramini, C. M. M., Santos, A. A. A., Polydoro, S. A. J., Sbardelini, E. T. B., Serpa, M. N. F. \& Natário, E. G. (2004). Construção e validação de uma escala sobre avaliação da vida acadêmica (EAVA). Estudos de Psicologia (Natal), 9, 259-268.

Zago, N. (2006). Do acesso à permanência no ensino superior: percursos de estudantes universitários de camadas populares. Revista Brasileira de Educação, 11, 226-370.

Recebido: 01/05/2013

Última revisão: 06/03/2015 Aceito: 11/03/2015 